\title{
A TIME SYNCHRONIZED HYBRID VEHICULAR AD HOC NETWORK OF ROADSIDE SENSORS AND VEHICLES FOR SAFE DRIVING
}

\author{
Dahlia Sam and V. Cyril Raj \\ Department of CSE, Dr M.G.R Educational and Research Institute University, Chennai, India
}

Recieved 2013-10-14; Recieved 2014-01-29; Accepted 2014-04-10

\begin{abstract}
VANET is a type of ad hoc network in which the moving vehicles act as nodes. There has been lot of research for using VANETs in many applications. One of the main applications is the use of VANETs to improve driving safety. In any safety related applications, the vehicular nodes have to constantly communicate with each other and the roadside equipments. For e.g., the roadside units sense real time information about road conditions, road blocks or animals straying on the road and passes the message to the approaching vehicles. The alert message enables the driver to take timely decisions in preventing accidents or delay. However there are two issues in the above system. One problem is that VANETs are subject to frequent network disconnections especially in low traffic areas. Due to this some events in the road may go undetected while the detected events may not be transmitted on time. The second issue it with maintaining a synchronized clock within the network. Only then the messages communicated between the nodes will be meaningful. To overcome the above issues and make the system more reliable we propose to include roadside wireless sensor nodes along with the vehicular nodes in the network. The roadside wireless sensor nodes can be deployed at fixed distances and communicate wirelessly with the vehicular nodes. They play an important role in keeping the network connected and guarantee message transmission. We also propose a Hybrid Clock Synchronization (HCS) algorithm to synchronize the clocks of all the nodes. This integrated network which is also time synchronized is called the Hybrid VANET (H-VANET). The proposed H-VANET was simulated and tested using GrooveNet. On comparing our system with the conventional VANET implementing RBS for synchronization, it was seen that our model has better performance and reliability.
\end{abstract}

Keywords: Hybrid VANET, H-VANET, Hybrid VANET-WSN, Hybrid Clock Synchronization (HCS)

\section{INTRODUCTION}

In today's world driving is becoming an indispensible part of everyone's life. The number of drivers on the road has been steadily increasing over the years. There are more vehicles on the road than ever before. This number is sure to keep increasing in future. With this, the need for a real time intelligent vehicle communication system has become very important. One promising technology of the future that focuses on this issue is the Vehicular Ad hoc Corresponding Author: Dahlia Sam, Department of CSE, Dr M.G.R Educational and Research Institute University, Chennai, India
Network (VANET). Researchers are working on using VANETs for applications like driving safety, intelligent speed control, lane changing, safe highway entry and exiting, timely warning during hard braking and accidents. All these aid to improve the safety of the highway system.

\subsection{Background}

Vehicular communication has become an important area of research in the past decade. There has been lot of study going on to develop an Intelligent Transport

\section{7}


System (ITS). The first form of vehicular communication that was proposed used optical laser or infrared laser (Fujii et al., 1995; Sasaki et al., 1994; Mizui et al., 1994). In this each vehicle can communicate with the vehicle directly in front of it and the one directly behind it in the same lane. This system has the drawback that each vehicle can communicate with only two vehicles. The communication is also very sensitive to the alignment of the vehicles and weather conditions like rain, fog or snow. Another method proposed was communication using Radio Frequency (RF) (Kremer et al., 1993; Valade, 1995). Here the vehicle can broadcast to all the vehicles in its range. Reservation ALOHA (R-ALOHA) protocol is used for medium access. Later in 1999, the Federal Communications Commission (FCC) allocated $75 \mathrm{MHz}$ of spectrum at 5.850-5.925 GHz for Dedicated Short Range Communications (DSRC) (Yue et al., 2009). The allotted frequency spectrum enabled wireless communication between vehicle-vehicle and vehicle-roadside beacons without central access point. This led to the development of VANETs and its related services. VANET can be defined as "computer network on wheels". It is a network with the moving cars as the mobile nodes (Yousefi et al., 2006; Chandrasekaran, 2007). These nodes communicate with each other as well as with the roadside equipments which are within ranges of 100 to $300 \mathrm{~m}$ based on IEEE 802.11 p standard.

\subsection{Applications}

In the recent years, there have been numerous applications that have been proposed to be developed on top of this VANET (Ho Ting Cheng et al., 2011; Festag et al., 2008; Qin et al., 2010; Nagappan, 2012). The potential applications of the vehicular networks can be divided into five main category: (1) Road analysis (2) Road safety (3) Traffic management (4) Infotainment (5) Post accident investigation. Before any of the other applications, VANETs can be used to predict and analyze traffic, environmental factors, animal dangers etc in a particular area. This data can be used to design roads, bridges, fences or pavements to withstand those predicted conditions. Road safety applications focuses on accident prevention and avoidance. These deal with giving real time alerts about road conditions, collision warning, smart navigation and merge assistance. Traffic management aims at improving road capacity, avoiding traffic congestion, taraffic light scheduling, intersection traffic management etc. Infotainment applications include on-the-road games, media streaming, digital billboards for advertisements, business mails etc. Finally, the RSUs in the VANET can continuously measure and store the happenings in the road which can be used later for forensic reconstruction or other post accident investigation In this study we specifically focus on improving driving safety using real time road information with the help of a time synchronized hybrid VANET.

\section{HYBRID VEHICULAR AD HOC NETWORK (H-VANET)}

\subsection{Motivation}

VANETs are currently only in research and has not yet been practically deployed. For any of the above mentioned applications to work, we need a minimum market penetration of equipped vehicles. An equipped vehicle should have an onboard laptop with embedded WiFi card and also meet some other requirements. A minimum market penetration of $10 \%$ of VANET equipped vehicles is needed to make this network a reality. For reaching this $10 \%$ in a period of 3 years, at least $50 \%$ of the newly produced cars should be VANET enabled i.e., it should support V2V and V2I communication (Yousefi et al., 2006). This value added vehicles are on their way to being introduced in the market as the technology has been theoretically proven to be effective and efficient. However, for the system to work, we also need a minimum number of roadside access points to be installed starting with national highways. The Road Side Units (RSUs) should also be well equipped, installed and maintained properly. The wide scale purchase, deployment and maintenance of the required infrastructure for such a system can be an expensive affair. It may not be practically feasible over the next few years. This major barrier for market penetration can be overcome by combining the low cost WSN with the VANETs.

A pure VANET consists of vehicular communication over multiple wireless hops but it may or may not include roadside access points. Even if roadside access points are deployed, it is only feasible to deploy them in some important highways due to its high cost. Hence any two consecutive RSUs may not be in the direct communication range of each other. Whenever there are enough vehicles on the road a network is formed between the vehicles and the roadside sensor. However, if the vehicles in the road are sparse, they may not be in the communication range of each other. This may commonly happen in remote roads or during low traffic hours. There may not be any vehicles on the road to sense an event. The vehicle will detect the event only when it is in the close vicinity, often when it is too late to take any decisions. Even if one vehicle detects the event and if there is no proper connectivity between the nodes, the collected information cannot be shared with the other vehicles. The alert message may not get passed on to the approaching vehicles. 
One of the solutions proposed for this problem is discussed by Fathima and Wahidabanu (2011). They have suggested the use of Delay Tolerant Networks (DTN) that operates on the principle of store-carry and forward routing. The messages are stored by the nodes until the next node hop is available for forwarding. Anggoro et al. (2013), has proposed combining probabilistic relay with AODV and AOMDV protocols. In a situation if the vehicle, due to its dynamic nature, moves out of the range of its next hop then obviously the transmission fails. Anggoro et al. (2013), has suggested that the adjacent vehicles can probabilistically relay unsuccessful transmissions. However the trade-offs in both the above proposals is the message delivery delay. V2V message communications may not be feasible when the vehicles in the road are sparse. The messages may not reach the destination on time to prevent the accident, which is a very crucial factor. Another method proposed is the use of Ariel remote sensing for highway incident detection (Kahaki et al., 2011). However, this method has been only $80 \%$ sucessful and is also expensive to implement.

A complementary cost effective solution to overcome these constraints in conventional VANETs is the Hybrid Vehicular Ad Hoc Network (H-VANET). In H-VANET, the VANET is integrated with the Wireless Sensor Nodes (WSN). Low cost wireless sensors are deployed in between two access points. The Hybrid VANET is more efficient in detecting the events ahead of time using the static roadside sensors. Thus H-VANETs provide a much reliable and cheaper solution. The sensor node can be deployed in curvy roads, tunnels, bridges easily. They can also be used to sense physical data like temperature, humidity, light, motion. The sensor nodes are battery powered and run for many months with a pair of AA batteries. Due to its ease of deployment and low cost, it can easily cover a wide geographic area.

\subsection{Advantages}

In this section, some practical examples that can happen in everyday life has been listed. In real life accidents can happen due to any of the understated factors (Khairunnisa et al., 2014). In all of these examples, it can be clearly seen that the presence of roadside sensors could make VANETs more effective.

\subsubsection{Road Factors}

The roads can become slippery as a result of rain or snow. The vehicle that has passed through the slippery route can send a message to the approaching vehicles.
This way the approaching vehicles can take precautionary steps or take an alternate route. A curvy or steep road ahead can be cautioned to the following vehicles by the front vehicle. The message reaches the other vehicles through the roadside sensors even if they are not in the direct communication range of each other.

There can also be a wide range of unexpected road blocks like an accident in the road or a fallen tree. The roadside sensors can prevent chain accidents by informing the situation ahead of time helping the driver take timely decisions.

\subsubsection{Environmental Factors}

In some places fogs cover the roads affecting visibility. The visibility can be reduced to 10-20 meters. Visibility is also reduced during night time and during rain. A pedestrian walking in the highway may not be visible to the driver. If the roadside sensors can sense a human in the road and pass the information to the approaching vehicles, pedestrian accidents could be avoided.

\subsubsection{Human Factors}

In practical life we may come across many other emergency situations. Kids playing in the backyard could accidently run into the roads. Similarly old age or handicapped persons trying to cross the road may not be able to see the approaching vehicles or make it to the other side quickly. In such cases if a roadside sensor could detect their presence and warn the vehicles beforehand, the drivers will have enough time to process the scenario and apply the brakes gradually.

The roadside sensor nodes also continuously detect the happenings in the road and store it within the sensor network. This may be useful in post accident investigations especially in hit and run cases (Festag et al., 2008).

\subsubsection{Animal Factors}

It is quite common for animals to keep roaming on the roads that can cause accidents (http://en.wikipedia.org/wiki/Deer-vehicle_collisions). In 2000 , out of 6.1 million collisions in US 247,000 crashes were deer-vehicle collisions. A sensor node in the $\mathrm{H}$ VANET could immediately detect an animal roaming in the road and pass the information to the approaching vehicles. The driver can slow down and drive cautiously when entering that route.

The advantages of a Hybrid VANET over a conventional VANET can be summarized in Table 1. 
Table 1. Advantages of H-VANET

\begin{tabular}{lll}
\hline Scenario & VANETs & H-VANETs \\
\hline Reliability & $\begin{array}{l}\text { Sometimes there may not be any vehicles } \\
\text { on the road to detect a particular event } \\
\text { Poor network connectivity in tunnels, } \\
\text { remote roads, hills and bridges }\end{array}$ & The roadside sensors will never miss an event \\
Network stability & $\begin{array}{l}\text { Network can get disconnected frequently } \\
\text { when the vehicles are sparse } \\
\text { The network exists only when vehicles } \\
\text { are present on the road. The events on } \\
\text { the road may go unnoticed }\end{array}$ & $\begin{array}{l}\text { Sensor nodes can be easily deployed in any } \\
\text { geographical locations } \\
\text { The sensor nodes help to keep the network } \\
\text { connected all the time }\end{array}$ \\
& & $\begin{array}{l}\text { Here the design is very flexible. We can } \\
\text { deploy a cloud of sensor nodes in places that } \\
\text { are more prone to dangerous events. Similarly } \\
\text { in safe roads where we do not need a constant } \\
\text { sensor node for monitoring, we do not have to } \\
\text { deploy them } \\
\text { Sensor node technology is less expensive and } \\
\text { well developed. This makes H-VANET a } \\
\text { more feasible alternative }\end{array}$ \\
\hline
\end{tabular}

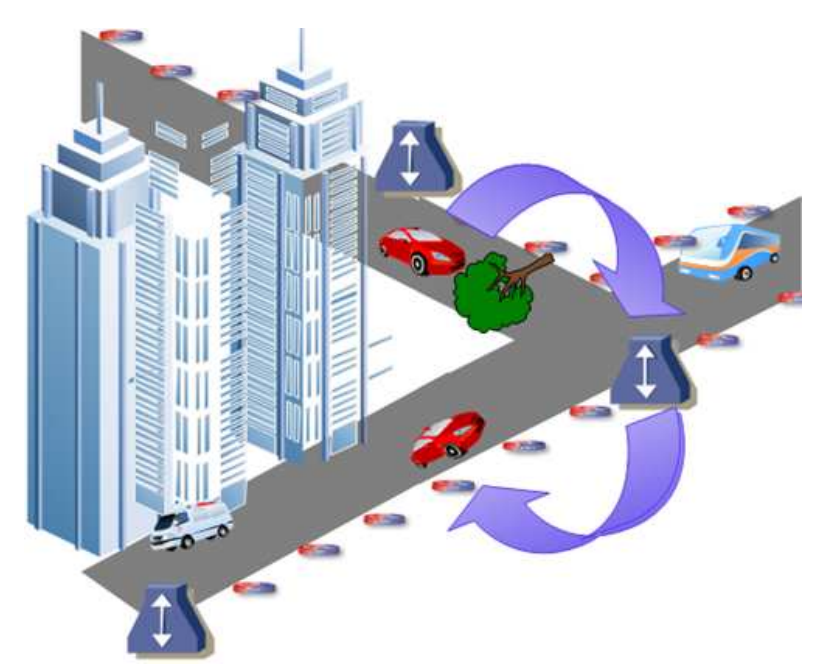

sensor information and transmits the aggregated data to the other RSUs. It also maintains the data in its local database and transfers it to the vehicle nodes when a vehicle comes in its communication range. Once a vehicle receives the data, it distributes the data to the other vehicles in a geographical location by the Geocast Protocol. The message is communicated to the drivers using some Driver Assistance System (DAS) (Singh, 2010). Maintaining the security of the communication messages is also important and is beyond the scope of this study. ANET security protocols have been discussed by (Chen et al., 2013; Pattnaik and Pattanayak, 2014).

The device (or on board unit) in the vehicle will have two interfaces: Embedded WiFi card (IEEE 802.11) that is used for communication with the other vehicles and a IEEE 802.15.4 (ZigBee) interface for communication with the RSUs. The sensor nodes communicate with each other and with the vehicle nodes using the IEEE 802.15.4 (ZigBee) communication interface. Similarly the RSUs also have 2 communication interfaces. RSUs and sensor nodes are deployed on both the sides of the road in a two way highway. There are fewer RSUs that are deployed at fixed distances. The sensor nodes are deployed in between two adjacent RSUs. The sensor nodes can sense and relay messages whereas the RSUs can also communicate with the vehicles. The optimal placement of the RSUs and sensor nodes has been discussed in (Rebai et al., 2012). IEEE 802.15.4 costs less, is more energy efficient and communicates over a small geographical area. Hence it is used in the sensor nodes. On the other hand, IEEE 802.11 used in the vehicle node is more expensive but it can transfer data over medium distances via multi hop communication.

tide wireless sensor nodes are divided into group each group is managed by a RSU. The RSU collects all 


\section{HYBRID CLOCK SYNCHRONIZATION (HCS)}

In any of the scenarios mentioned in the previous section (2.2), the communication between vehicles is mandatory. The most important factor in these communications is that the clock times of the different nodes have to be synchronized. Suppose a caution message is sent by one vehicle at time 10:00. The message is delivered to the vehicle directly following it. Suppose the time of message delivery in the destination vehicle is 9:59. The destination vehicle will not be able to take any decision based on the message. The caution message becomes meaningless because the time in both the vehicles is not synchronized. A perfectly synchronized time is also necessary for taking decisions based on messages sent by multiple vehicles. In many similar situations, varying degree of clock precision is required based on the application.

The main aim of clock synchronization is to provide a common reference point for all the nodes connected in a network. Synchronization in Hybrid VANET can be done in two ways: Centralized synchronization and decentralized synchronization (Sourour and Nakagawa, 2008; Shizhun et al., 2010). The centralized approach makes use of a GPS to synchronize with the global time. In the decentralized approach any node can initiate the synchronization process. The different decentralized approaches that have been proposed are: 1. Time signal method-Here, every node transmits a timing signal continuously. The phase offset is calculated by comparing with the received signals. 2. Pulse based method-here every node periodically transmits a pulse. Each node corrects its own clock based on the incoming pulse. e.g.:- Mutual synchronization, slot synchronization 3. Clock offset method-Here every node transmits its clock time with its neighbors. The nodes calculate the clock offset by comparing its local clock with the neighboring nodes clocks. e.g.:- Reference Broadcast Synchronization (RBS).

\subsection{Algorithm}

In this study, we propose a Hybrid Clock Synchronization (HCS) protocol for time synchronization in a hybrid VANET-WSN network. The algorithm is very robust, scalable and is not affected by the frequent topology changes that are a characteristic of VANETs. It aims to synchronize the RSU with the sensor nodes and the vehicle nodes within its coverage area. Each vehicle has its own unique ID, a list of nodes that it is synchronized with and a list of neighboring nodes within its coverage area. The neighbors will include the vehicle nodes, RSUs and sensor nodes. The vehicle nodes maintain its neighbor list by periodically broadcasting its unique ID. The size of the synchronized members is called the synch scale.

The synchronization process takes place in the following steps:

Step 1: Watch for Initialization

In our Hybrid-VANET system, the RSUs or any vehicle node could initiate the synchronization process. The RSUs can initiate the synchronization process at fixed intervals. This interval of time is referred to as a Synchronization Interval (SI). In places where there are no RSUs deployed or in cases when the RSU is down, any vehicle can randomly initiate the synchronization process. In either case, the synchronization process can be initiated only if there hasn't been an initialization message in one full synchronization interval i.e., no other node has initiated the process already. This will prevent multiple synchronization attempts by different nodes.

\section{Step 2: Synch initialization.}

If a node has already initiated the synchronization then the other nodes cooperate and pass on the required information. The initiator, either the RSU or any vehicle will now broadcast a Collection Message (CM) to all the neighbors in its transmission range. The collection message contains a collection request, all the neighbors IDs and a reply sequence for all the neighbors to avoid reply collisions. As soon as the other nodes receive the Collection Message it will know that it doesn't have to initiate the synchronization in that cycle.

Step 3: Send reply message.

On receiving the collection message, the node will check the reply sequence and find its time slot. It will then set a timer. When the time expires it will send a Reply Message (RM) to the initiator. The reply message contains the synch scale, unique ID and the time difference of the node. The time difference is the deviation of the nodes clock with respect to a standard clock e.g., GMT. The format of the reply message is shown in Fig. 2.

\begin{tabular}{|l|l|c|}
\hline Node ID & Synch scale & $\begin{array}{c}\text { Clock } \\
\text { deviation }\end{array}$ \\
\hline
\end{tabular}

Fig. 2. Reply message format 
Step 4: Reply collection.

The initiator receives the Reply Messages from all the neighbors. The initiator waits for a time period, $\mathrm{T}_{\text {reply }}$ to get the reply message from all its neighbors Equation (1):

$$
\mathrm{T}_{\text {reply }}=[(\mathrm{N}+1) * \mathrm{R}]
$$

Where:

$\mathrm{N}=$ The number of neighbors

$\mathrm{R}=$ The duration of one reply message

Step 5: Selecting the synchronizer.

The initiator will compare the synch scales of all the neighbors with its own synch scale. If any vehicle node has a higher scale than its own synch scale, then that becomes the synchronizer. The initiator will then send a message to that node informing that it is the new synchronizer. It will also send a list of all the vehicles' IDs. On the other hand, if the initiator itself is the node with the highest synch scale then it will continue and take up the role as synchronizer.

\section{Step 6: Synchronization.}

The synchronizer will edit its synch scale by updating the list of synchronized group members. It will then send a Clock Adjustment Message to all its group members. The message consists of the synchronizers time difference and all receivers IDs.

\section{Step 7: Clock adjustment.}

Finally the individual nodes will adjust their own clock and also its synch scale.

\section{EXPERIMENTAL RESULTS}

\subsection{Theoretical Results}

An important performance metric for a clock synchronization algorithm is the total time taken for the synchronization process. This is because the transmission time from the VANET to the WSN is very critical. The transmission range of the RSUs is between $30-80 \mathrm{~m}$. Suppose the transmission range of the sensor is $30 \mathrm{~m}$ and the vehicle is assumed to travel at an average speed of 70 $\mathrm{km} / \mathrm{hr}$. Under these conditions the vehicle will be in the transmission range of the RSUs for less than 1.5 se. All the message communications for the whole synchronization process has to take place within this time. The relative timing scale for each operation of HCS is given in Fig. 3.

Suppose SI = Synchronization Interval

$$
\begin{array}{ll}
\mathrm{CH} & =\text { Computer Handling } \\
\text { MT } & =\text { Message Transmission } \\
\mathrm{N} & =\text { No of neighbors } \\
\mathrm{R} & =\text { Reply time slot }
\end{array}
$$

The total time, $\mathrm{T}_{\text {total }}$, taken for one synchronization cycle of the HCS is given by Equation 2:

$\mathrm{T}_{\text {total }}=\mathrm{SI}+4 * \mathrm{CH}+3 * \mathrm{MT}+(\mathrm{N}+1) * \mathrm{R}$

Let us assume SI as $100 \mathrm{~ms}$, CH between $5-30 \mathrm{~ms}$, MT between 10-100 ms and R as $100 \mathrm{~ms}$. Substituting N $=10$ in the above equation, we get the total time taken for one synchronization cycle, $T_{\text {total }}$ between $1250 \mathrm{~ms}$ and $1620 \mathrm{~ms}$. So if the number of vehicles in the road is less, the probability of a vehicle to be in the range of the RSU reduces. But with the above calculation we see that for any number of vehicles below 10, there is enough time for the vehicles in the transmission range of the RSU. Suppose if the number of vehicles is increased as $\mathrm{N}=25$ to consider traffic jam condition. In this case the total time taken $\mathrm{T}_{\text {total }}$ is between 2750 and $3120 \mathrm{~ms}$.

During high traffic conditions, there is a higher probability that at least one vehicle in a group to be synchronized is in the range of the RSU. This guarantees enough time for communication of synchronization messages between the vehicle nodes and the sensor nodes.

The inequality between the expected number of retransmissions and the packet loss ratio is given by Equation 3:

$\sum_{i=1}^{n} \frac{p_{i}}{1-p_{i}} \leq r$

Where:

$\mathrm{n}=$ Number of sensors

$P_{i}=$ Packet loss ratio

$\mathrm{r}=$ Number of retransmissions

\subsection{Field Tests}

We conducted a set of experiments in a large parking lot to test how efficiently the message is being delivered to all the nodes. The system that was implemented had 3 components-the Road Side Unit (RSU), normal sensor nodes and vehicular nodes. The vehicle nodes are implemented by fixing a laptop in the vehicle with an attached telosb mote.

\begin{tabular}{|l|l|l|l|l|l|l|l|l|}
\hline SI & $\mathrm{CH}$ & $\mathrm{MT}$ & $(\mathrm{N}+1) \mathrm{R}$ & $\mathrm{CH}$ & $\mathrm{MT}$ & $\mathrm{CH}$ & $\mathrm{MT}$ & $\mathrm{CH}$ \\
\hline
\end{tabular}

Fig. 3. Time sequence for one synchronization cycle 
Table 2. Prototype testing platform

\begin{tabular}{lll}
\hline & Vehicle nodes & Sensor nodes \\
\hline Processor & 64 bits MIPS, 266 MHz & 16 bits MCU, $8 \mathrm{MHz}$ \\
Memory & $512 \mathrm{MB}$ & $10 \mathrm{~KB}$ RAM \\
External memory & $16 \mathrm{MB}$ flash & $48 \mathrm{~KB}$ flash \\
Microcontroller & & MSP430 \\
Power supply & $5.4-22 \mathrm{VDC} @ 400 \mathrm{~mA}$ & $3 \mathrm{VDC} @ 25 \mathrm{~mA}$ \\
Transceiver & $250 \mathrm{kbit} / \mathrm{s} 2.4 \mathrm{GHz}$ IEEE 802.15.4 & chipcon wireless transceiver \\
Network interface & IEEE $802.11 \mathrm{p}$ & IEEE 802.15.4 \\
Connectors & UART, USB, MOST, VICS & UART, SPI, I2C \\
Antenna & External, Omni-directional & Directional or omni-directional \\
Operating system & Linux 2.6 & TinyOS \\
\hline
\end{tabular}

Table 3. Average time taken for an alert message to reach all the nodes in a group

\begin{tabular}{llc}
\hline $\begin{array}{l}\text { Number } \\
\text { of vehicles }\end{array}$ & $\begin{array}{l}\text { Velocity } \\
(\mathrm{km} / \mathrm{hr})\end{array}$ & $\begin{array}{l}\text { Average message } \\
\text { delivery time }(\mathrm{ms})\end{array}$ \\
\hline 5 & 15 & 660 \\
& 25 & 720 \\
10 & 15 & 850 \\
& 25 & 910 \\
15 & 15 & 960 \\
& 25 & 1030 \\
20 & 15 & 1120 \\
& 25 & 1250 \\
\hline
\end{tabular}

Table 4. Simulation parameters

\begin{tabular}{ll}
\hline Highway length & $18900 \times 20 \mathrm{~m}$ \\
\hline Number of sensor nodes & 200 \\
Distance between two sensors & $80 \mathrm{~m}$ \\
Transmission range of sensor node & $100 \mathrm{~m}$ \\
Transmission range of vehicle nodes & $250 \mathrm{~m}$ \\
Average packet loss ratio & $15 \%$ \\
Synchronization Interval & $600 \mathrm{~ms}$ \\
Time between two events & $5-7 \mathrm{~min}$ \\
Simulation time & $60 \mathrm{~min}$ \\
\hline
\end{tabular}

The regular sensors and the access points are implemented as Telosb motes with mounted sensors. The sensors that we use here are long range WiEye Passive Infrared (PIR) sensors. It has a wide detection cone of $90-100^{\circ}$, a detection range of $20-30$ feet for human presence and 50-150 feet detection range for vehicles depending on the size.

The WiEye has a visual light sensor and acoustic sensor that improves the detecting ability of the PIR sensor. The WiEye sensor is directly plugged in to the TelosB motes. For our experiment, 20 Telosb motes were deployed along one side of the road. The distance between the motes was set as $40 \mathrm{~m}$. Every 10th mote was set as a RSU. The test lasted for 30 min. Vehicles were driven by volunteers at different velocities from one end to another. The detailed system specifications are listed in Table 2.

Whenever a vehicle spots an obstacle it immediately informs the nearby RSU and the vehicles in its range. For the roadside sensors, every object that enters its transmission range will be detected as an event. This may include a vehicle itself. In order to avoid this we made the following assumption. A normal vehicle on the road would travel at a minimum speed of $15 \mathrm{~km} / \mathrm{hr}$. In this case it will take about 7.2 se for the vehicle to pass the transmission range of the RSU. So the sensors will wait for $7.2 \mathrm{~s}$ after it detects an obstacle. If the obstacle still exists in the communication range after $7.2 \mathrm{sec}$, an alert message is communicated to the neighboring RSU and the approaching vehicles.

The test was conducted in a parking lot and the maximum speed of the test vehicles was set as $25 \mathrm{~km}$ $\mathrm{h}^{-1}$ for safety reasons. First a set of 5 volunteers were asked to drive through the parking lot. An event was generated at a random time by throwing a dummy doll in the parking lot. The time taken for the sensors to detect the event and communicate it with the vehicles in our study area was recorded.

The results obtained show that the message gets delivered to all the vehicles within few seconds, thus enabling the drivers to take decisions accordingly. The times taken for the message to be communicated in different scenarios are noted. The values are tabulated below in Table 3. When the numbers of volunteers were increased, the average message delivery time also increased. This may be accounted to increased number of message delivery destinations. There is also more packet loss due to higher interference and therefore more number of retransmissions. 


\subsection{Simulations}

We simulated our proposed H-VANET system using the GrooveNet simulator (GrooveNet, 2012). It is a very practically useful simulator because of its hybrid nature i.e., it enables communication between the simulated nodes and the real vehicles. The HCS algorithm was evaluated under different scenarios and the results are presented below. The parameters that were fixed in our simulation are shown in Table 4.

First, the performance of the HCS protocol was evaluated under different traffic conditions. The algorithm is very stable under heavy traffic conditions like traffic jams, normal traffic and under low traffic conditions like remote highways. The results are shown in Fig. 4.
The results show that whenever new vehicles enter the group they are synchronized to the existing group. The number of vehicles that have synchronized clock steadily increases as new vehicles enter the group. In contrast the existing algorithms, e.g.: RBS have to restart the synchronization process whenever a vehicle with a different time enters the group. Thus our synchronization algorithm performs better than RBS. Secondly, the effect of the vehicle speed with the speed of convergence was observed. The convergence speed is the time taken for the clocks of the different nodes to get synchronized. It was noted (Fig 5) that when the average speed of all the vehicles increases the time taken for convergence is high.

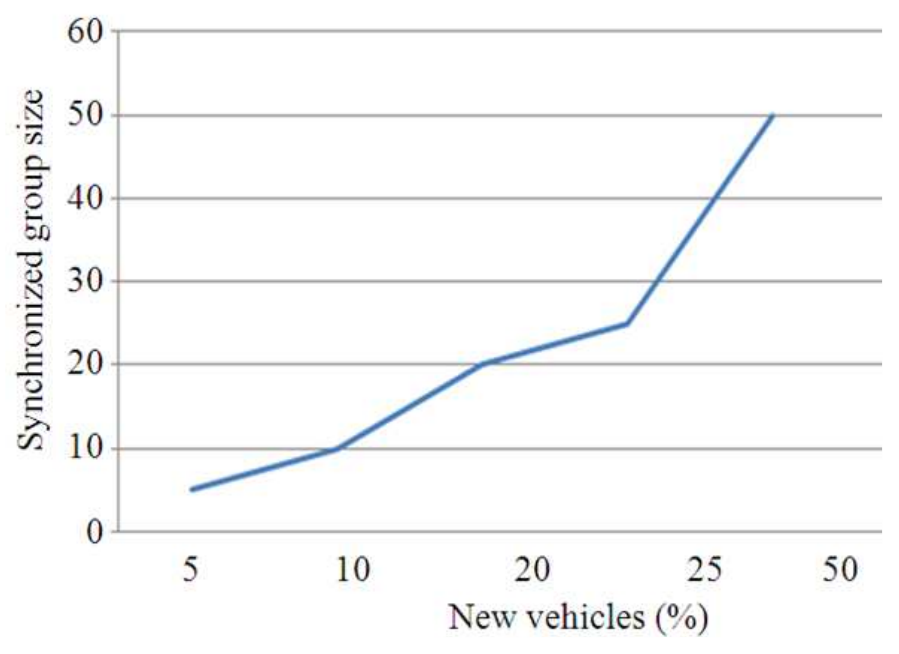

Fig. 4. Stability of the algorithm when new vehicles enter the group

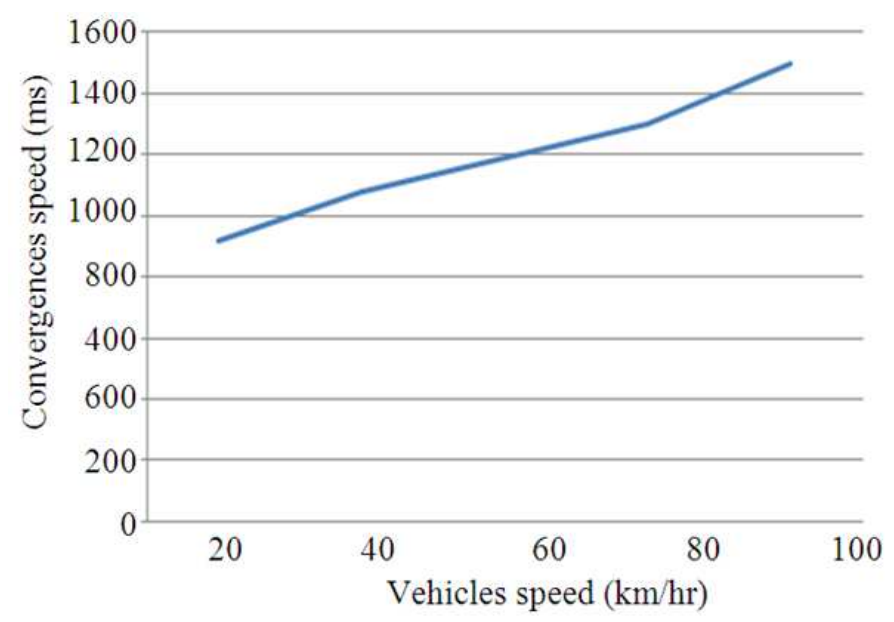

Fig. 5. Time taken for synchronization with respect to the average vehicle speed 


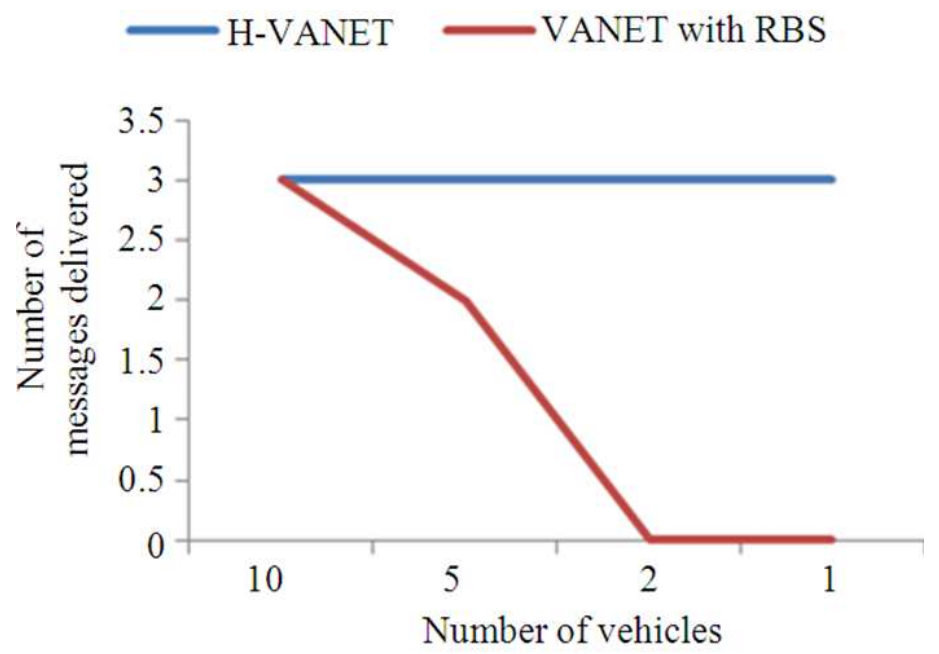

Fig. 6. Number of messages delivered within the acceptable time window

\section{DISCUSSION}

The H-VANET was compared with a normal VANET with RBS algorithm. The systems were compared considering some random low traffic scenarios. This is because in VANETs, low traffic scenarios face frequent network disconnections. Some of the typical situations when there are very few vehicles on the road include remote highways, tunnels, hilly roads and night time. The message passed between the vehicles will be useful and meaningful only if the message is delivered early enough for the driver to take an appropriate decision. The time between the earliest time and the latest time that a message could be delivered such that the driver is able to perceive and react to the message is referred to as the "Acceptable time window". The message delivered before or after this window becomes useless. We have analyzed the number of messages delivered within this acceptable time window for H-VANET and the conventional VANET under different traffic conditions. It can be seen that the conventional VANET with RBS fails to deliver the message when the number of vehicles on the road reduces. The H-VANET however is consistent and obviously more reliable as seen in Fig. 6.

\section{CONCLUSION}

In this study, we have proposed a novel idea to make the proposed idea of VANETs more reliable. All the foreseen applications of VANETs require the detection of real time events as well as timely communication of the detected events to the vehicles. Due to the unpredictable number of nodes and the fast changing topology of VANETs, it is sometimes impossible to detect and communicate the events on time. The new HVANET architecture that we have proposed integrates sensor nodes with the vehicular nodes to form a hybrid network. The sensor networking technology is well developed, very cost effective and efficient in detecting real time events in the roads. Integrating WSN with the VANET leverages the overall system. The static sensors of the H-VANET that are deployed in the roadside, assure that none of the events on the road go undetected. It also assures constant connectivity of the network irrespective of the number of vehicles present in the road. We have also proposed a Hybrid Clock Synchronization (HCS) algorithm to synchronize the clocks of the sensor nodes, roadside access points and the vehicular nodes. This is very important as the communicated messages are meaningful only if the clocks are time synchronized. The HCS algorithm has been simulated using a very reliable simulation platform and its performance has been tested under various conditions. The results show that HCS is a very stable protocol under both high node mobility and under low traffic conditions. We conclude that the H-VANET system together with the HCS proves to be a very attractive, cost efficient and reliable networking infrastructure for supporting all future vehicular applications. 
However we have simulated and experimented with the limited assumptions and implementations, the real traffic scenario has many more factors to be considered. The traffic regulations and patterns vary from country to country and also from region to region. Considering all the factors are beyond the scope of this study. Moreover, there may be some extreme situations where the time window between the alert message and the drivers' reaction may not be insufficient to prevent an accident. The reaction times of the drivers vary. Also, a distracted driver may overlook the alert message. To address these, our future work will include automatic controlling of vehicles. A system can be designed to automatically receive the alert message and take appropriate action like applying brakes or slowing down.

\section{REFERENCES}

Anggoro, R., T. Kitasuka, R. Nakamura and M. Aritsugi, 2013. Performance evaluation of probabilistic relay in ad hoc on-demand distance vector and ad hoc ondemand multipath distance vector under highly dynamic environments. J. Comput. Sci., 9: 905-921. DOI: 10.3844 jessp.2013.905.921

Chandrasekaran, G., 2007. VANETs: The networking platform for future vehicular applications. Department of Computer Science.

Chen, C.L., I.C. Chang, C.H. Chang and Y.F. Wang, 2013. A secure ambulance communication protocol for VANET. Wireless Personal Commun., 73: 11871213. DOI: $10.1007 / \mathrm{s} 11277-013-1273-y$

Cheng, H.T., H. Shan and W. Zhuang, 2011. Infotainment and road safety service support in vehicular networking: From a communication perspective. Mechanical Syst. Signal Proces., 25: 2020-2038. DOI: 10.1016/j.ymssp.2010.11.009

Fathima, G. and R.S.D. Wahidabanu, 2011. Integrating buffer management with epidemic routing in delay tolerant networks. J. Comput. Sci., 7 : 1038-1045. DOI: 10.3844/jcssp. 2011.1038 .1045

Festag, A., A. Hessler, R. Baldessari, L. Le and W. Zhang et al., 2008. Vehicle-to-vehicle and road-side sensor communication for enhanced road safety.

Fujii, H., K. Seki and N. Nakagata, 1995. Experimental research on protocol of inter-vehicle communication for vehicle control and driver support. Proceedings of the 2nd World Congress on Intelligent Transport System, Nov. 9-11, Yokohama, Japan, pp: 1600-1605.
GrooveNet, 2012. GrooveNet hybrid-network simulator for vehicular networks groovenet hybrid-network simulator for vehicular networks. GrooveNet.

Kahaki, S.M. Mousavi and M.J. Nordin, 2011. Highway traffic incident detection using high-resolution aerial remote sensing imagery. J. Comput. Sci., 7: 949953. DOI : 10.3844/jcssp.2011.949.953

Kremer, D.W., D. Hubner, S. Holf, T. Benz and W. Schafer, 1993. Computer-aided design and evaluation of mobile radio local area networks in RTI/IVHS environments. IEEE J. Select. Areas Commun., 11: 406-421. DOI: 10.1109/49.219554

Mizui, K., M. Uchida and M. Nakagawa, 1994. Vehicleto-vehicle 2-way communication and ranging system using spread spectrum technique: Proposal of double boomerang transmission system. Proceedings of the IEEE Conference on Vehicle Navigation and Information System, Aug. 31-Sept. 2, Yokohama, Japan, pp: 153-158. DOI: 10.1109/VNIS.1994.396848

Pattnaik, O. and B.K. Pattanayak, 2014. Security in vehicular ad hoc networks. 11: 337-346.

Qin, H., Z. Li, Y. Wang, X. Lu and W. Zhang et al., 2010. An integrated network of roadside sensors and vehicles for driving safety: Concept, design and experiments. Proceedings of the IEEE International Conference on Pervasive Computing and Communications, Mar. 29-Apr. 2, IEEE Xplore Press, Mannheim, pp: 79-87. DOI: 10.1109/PERCOM.2010.5466988

Rebai, M., L. Khoukhi, H. Snoussi and F. Hnaien, 2012. Optimal placement in hybrid VANETs-sensors networks. Proceedings of the Wireless Advanced, Jun. 25-27, IEEE Xplore Press, London, pp: 54-57. DOI: 10.1109/WiAd.2012.6296567

Sasaki, I., T. Hirayama and T. Hatsuda, 1994. Vehicle information network based on inter-vehicle communication by laser beam injection and retro reflection techniques. Proceedings of the IEEE Vehicle Navigation and Information System Conference, Aug. 31-Sept. 2, IEEE Xplore Press, Yokohama, Japan, pp: 165-168. DOI: 10.1109/VNIS.1994.396846

Shizhun, W., A. Pervez and M. Nekovee, 2010. Converging time synchronization algorithm for highly dynamic Vehicular Ad hoc Networks (VANETs). Proceedings of the 7th International Symposium on, Communication Systems Networks and Digital Signal Processing, Jul. 21-23, IEEE Xplore Press, Newcastle upon Tyne, pp: 443-448. 
Singh, D., 2010. Hybrid Auditory Based Interaction Framework for Driver Assistance System. J. Comput. $\quad$ Sci., $\quad$ : $1499-1504$. 10.3844/jcssp.2010.1499.1504

Sourour, E.E.A. and M. Nakagawa, 2008. Mutual decentralized synchronization for intervehicle communication. Alexandria University and Keio University.

Valade, J.M., 1995. Vehicle to vehicle communications: Experimental results and implementation perspectives. Proceedings of the 2nd World Congress on Intelligent Transport Syst. Nov. 9-11, Yokohama, Japan, pp: 1606-1613.
Yousefi, S., M.S. Mousavi and M. Fathy, 2006. Vehicular Ad Hoc Networks (VANETs): Challenges and perspectives. Proceedings of the 6th International Conference on ITS Telecommunications, Jun. 21-23, IEEE Xplore Press, Chengdu, pp: 761-766. DOI: 10.1109/ITST.2006.289012

Yue, L., B. Jun and J. Yang, 2009. Research on vehicular ad hoc networks. Proceedings of the Control and Decision Conference, Jun. 17-19, Guilin, pp: 44304435. DOI: 10.1109/CCDC.2009.5192343 